\title{
Outcomes of radiation therapy for resectable MO gastric cancer
}

\author{
Weipeng Gong ${ }^{1}$, Hongwei Zhao ${ }^{1}$, Shanshan Liu ${ }^{2}$, Jie Guan ${ }^{1}$, Xin Liu ${ }^{1}$, Qingsheng \\ Hou ${ }^{1}$, Zhenyu Zhu ${ }^{1}$ and Hongliang Guo ${ }^{1}$ \\ ${ }^{1}$ Department of Surgical Oncology, Shandong Cancer Hospital Affiliated to Shandong University, Jinan, Shandong Province, \\ 250117, China \\ ${ }^{2}$ Department of Radiation Oncology, Shandong Cancer Hospital Affiliated to Shandong University, Jinan, Shandong Province, \\ 250117, China
}

Correspondence to: Hongliang Guo, email: hliangbb@163.com

Keywords: surgery, gastric cancer, radiation therapy, resectable without distant metastases

Received: April 25, $2017 \quad$ Accepted: August 28, 2017

Published: November 03, 2017

Copyright: Gong et al. This is an open-access article distributed under the terms of the Creative Commons Attribution License 3.0 (CC BY 3.0), which permits unrestricted use, distribution, and reproduction in any medium, provided the original author and source are credited.

\section{ABSTRACT}

Background: The role of radiaotion therapy in resectable gastric cancer patients without distant metastases remains controversial. This retrospective analysis was performed to identify whether resectable gastric cancer patients without distant metastases might benefit from radiation.

Results: The results of the Kaplan-Meier analysis and log-rank test showed that a total of 3309 patients had a MST of 29.0 months, a 1-year survival rate of $74.7 \%$, and a 3-year survival rate of $45.5 \%$. Among them, the MST of the "RPS" group and the "RAS" group were significantly longer compared with that of the "No Radiation"

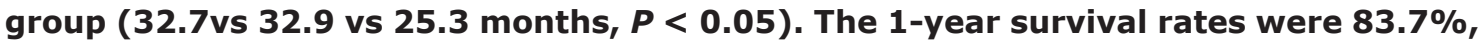
$83.5 \%$ and $65.6 \%$ for the "RPS", "RAS" and "No radiation" groups, respectively $(P<0.05)$ and the 3 -year survival rates were $52.6 \%, 63.6 \%$ and $44.9 \%$, respectively $(P<0.05)$. The multivariate Cox proportional hazard regression analysis showed that radiation was an independent prognostic factor.

Materials and Methods: A total of $\mathbf{5 7 4 4}$ patients from the SEER database who were initially diagnosed with histologically confirmed gastric cancer without distant metastases from 2010 to $\mathbf{2 0 1 3}$ were included. Patients were divided into three groups as follows: patients who underwent radiation after surgery ("RAS" group), patients who underwent radiation prior to surgery ("RPS" group) and patients who did not undergo radiation ,only surgery performed ("No radiation'"group).

Conclusions: This retrospective analysis demonstrated that "RPS" or "RAS"alone were independent prognostic factors for survival improvement in selected gastric cancer patients without distant metastases.

\section{INTRODUCTION}

Gastric cancer (GC) is the fourth most common type of cancer globally and the main cause of cancer-related mortality worldwide, specifically in Asian countries [1, 2]. An estimated 26,370 people will be diagnosed of gastric cancer and 10,730 people will eventually die of gastric cancer in the United States, in 2016 [3]. More than 70\% of cases occur in developing countries, Eastern Asia (mainly in China) occupies half of the world total cases [4]. The survival of early primary gastric cancer patients has improved because of early tumor detection, curative surgical resection and adjuvant therapy. However, gastric cancer is often diagnosed at an locally advanced stage [5]. Complete resected with D2 lymphadenectomy is widely regarded as the standard of care [6]. However, even after D2 gastrectomy and effective adjuvant chemotherapy, locoregional recurrence and distant metastasis are remarkable problems, and the survival time is usually unsatisfactory. Researchers have begun to seek and explore new and more effective treatment options for locally advanced gastric cancer patients without distant 
metastases. The Intergroup 0116 (INT 0116) study suggested that adjuvant chemoradiotherapy after curative surgery Improved survival outcome [7] was reconfirmed in several studies $[6,8,9]$.

Radiation therapy is used more commonly in treatment of gastric cancer at present [10]. However, in Eastern Asia countries D2 gastrectomy is accepted as a standard surgical procedure, adjuvant RT is not commonly given to completely resected patients $[11,12]$. However lacking sufficient evidence from large randomized trials, adjuvant RT after D2 dissection remained controversy. In this study, we retrospectively analyzed whether gastric cancer patients without distant metastases might benefit from postoperative radiation therapy.

\section{RESULTS}

\section{Patient characteristics}

A total of 3309 eligible patients were included: 623 (18.8\%) patients underwent radiation prior to surgery ("RPS" group), 1053 (31.8\%) patients underwent radiation after surgery ("RAS" group). A total of 2766 (48.2\%) patients were over 65 years old and $2144(64.8 \%)$ were male. Patient characteristics and demographics are summarized in Table 1.

\section{Survival analyses}

The results of the Kaplan-Meier analysis and logrank test showed that a total of 3309 patients had a MST of 29.0 months, a 1-year survival rate of $74.7 \%$, and a 3 -year survival rate of $45.5 \%$. Among them, the MST of the "RPS" group and the "RAS" group were significantly longer compared with that of the "No Radiation" group (32.7vs 32.9 vs 25.3 months, $P<0.05$ ). The 1 -year survival rates were $83.7 \%, 83.5 \%$ and $65.6 \%$ for the "RPS", "RAS" and "No radiation" groups, respectively $(P<0.05)$ and the 3-year survival rates were $52.6 \%, 63.6 \%$ and $44.9 \%$, respectively $(P<0.05)$.

\section{Outcomes of the different subgroups}

We compared the survival benefit of patients according to the subgroups, which accounted for age, gender, race, grade, T-stage, N-stage and AJCC tumor stage, by Kaplan-Meier analysis and log-rank test. Among the different subgroups, the survival benefits of patients in the "RPS" and "RAS" groups were better than those seen in patients in the "No radiation" group (Table 2). Specifically, in the age, race, T2, T3, T4a, T4b, IIIA, IIIB, Grade, N1, N2, N3, AJCC tumor stage, the results showed that the survival improvement of patients in the "RPS" and "RAS" groups was significantly higher compared with that of patients in the "No Radiation" group. Moreover, patients with stage IIB cancer in the "RAS" group had an increased survival benefit compared with those in the "RPS" group $(p<0.05)$. However, among those with stage IIIA, IIIB, IIIC cancer, no statistically significant differences were found in the MST, the 1-year or the 3-year survival rate among the "RPS" and "RAS" groups $(p>0.05)$ (Figure 3).

The superiority in the "RAS" groups was significantly higher compared with the "No radiation" group in the IIB, T4a, T4b, N0, and N1 subgroups. However, no statistically significant differences were found among"RPS" group compared with the "No radiation" group.

\section{Multivariate analyses for survival}

The multivariate Cox proportional hazard regression analysis showed that radiation was an independent prognostic factor ("RPS", hazard ratio (HR) $=0.797,95 \%$ confidence interval (CI) 0.650-0.977, $p<0.05$; "RAS", $\mathrm{HR}=0.515,95 \%$ CI $0.448-0.592, p<0.001)$. We also analysed all the aforementioned factors in the subgroups and found that age, race, N-stage, T-stage and tumor stage were also independent prognostic factors (Table 3, Figures 1, 2).

\section{Prognostic factors associated with radiation}

The multivariate Cox proportional hazard regression analysis showed that in the "RPS" group, age was independent prognostic factors, while in the "RAS" group, race, tumor stage and grade were independent prognostic factors (Table 4, Figure 4).

\section{DISCUSSION}

In recent years, radiation therapy (RT) for resectable gastric cancer patients without distant metastases has remained disputed [13]. In our study, we evaluated which locoregional subsites benefited most from adjuvant RT combination therapy among gastric cancer patients without distant metastases, who had undergone complete resection of gastric cancer. We found that adjuvant RT significantly prolonged survival time in completely resected gastric cancer without distant metastases patients, especially patients with LN metastasis (N1, $\mathrm{N} 2, \mathrm{~N} 3)$, primary tumor invasion $(T 2, T 3, \mathrm{~T} 4 \mathrm{a}, \mathrm{T} 4 \mathrm{~b})$ or locally advanced (IIIA,IIIB,IIIC).The multivariate Cox proportional hazard regression analysis showed that radiation was an independent prognostic factor ("RPS", hazard ratio $(\mathrm{HR})=0.797,95 \%$ confidence interval $(\mathrm{CI})$ $0.650-0.977, p<0.05$; "RAS", HR $=0.515,95 \%$ CI $0.448-0.592, p<0.001)$.

In a review based on 9 studies, Valentini et al. reported that the 5-year survival of resectable gastric cancer patients statistically significant benefit with the addition of RT [14]. Recently, SUNG KIM et al. 
Table 1: The characteristics of patients with gastric cancer without distant metastases

\begin{tabular}{|c|c|c|c|c|c|}
\hline Characteristic & $\begin{array}{l}\text { Overall no. }(\%) \\
\qquad(N=3309)\end{array}$ & $\begin{array}{l}\text { Radiation prior } \\
\text { to surgery }\end{array}$ & $\begin{array}{c}\text { Radiation } \\
\text { after surgery }\end{array}$ & No radiation & $p$ \\
\hline Age at diagnosis (y) & 3309 & $623(18.8)$ & $1053(31.8)$ & $1633(49.4)$ & $<0.001$ \\
\hline$\leq 65$ & $2766(48.2)$ & $421(24.1)$ & $638(36.5)$ & $691(39.5)$ & \\
\hline$<65$ & $2978(51.8)$ & $202(13.0)$ & $415(26.6)$ & $942(60.4)$ & \\
\hline Sex, $N(\%)$ & 3309 & & & & $<0.001$ \\
\hline Male & $2144(64.8)$ & $511(23.8)$ & $647(30.2)$ & $986(40.6)$ & \\
\hline Female & $1165(35.2)$ & $112(9.6)$ & $406(34.8)$ & $647(55.5)$ & \\
\hline Race, $N(\%)$ & 3289 & & & & $<0.001$ \\
\hline White & 2265 (68.9) & $558(24.6)$ & $613(21.7)$ & $1094(48.3)$ & \\
\hline Black & $386(11.7)$ & $18(4.7)$ & $169(43.8)$ & $199(51.6)$ & \\
\hline Other & $638(19.4)$ & $46(7.2)$ & $265(41.5)$ & $327(51.3)$ & \\
\hline T-stage $N(\%)$ & 3284 & & & & $<0.001$ \\
\hline T1a & $19(0.6)$ & $6(31.6)$ & $3(15.8)$ & $10(52.6)$ & \\
\hline $\mathrm{T} 1 \mathrm{~b}$ & $57(1.7)$ & $7(12.3)$ & $21(36.8)$ & $29(50.9)$ & \\
\hline $\mathrm{T} 2$ & $239(7.3)$ & $61(25.5)$ & $77(32.2)$ & $101(42.3)$ & \\
\hline $\mathrm{T} 3$ & $1795(54.7)$ & $499(27.8)$ & $515(28.7)$ & $781(43.5)$ & \\
\hline $\mathrm{T} 4 \mathrm{a}$ & 887 (27) & $22(2.5)$ & $342(38.6)$ & $523(59.0)$ & \\
\hline $\mathrm{T} 4 \mathrm{~b}$ & $287(8.7)$ & $19(6.6)$ & $91(31.7)$ & $17(61.7)$ & \\
\hline Tumor grade $N(\%)$ & 3160 & & & & $<0.001$ \\
\hline Well differentiated; Grade I & $74(2.3)$ & $26(35.1)$ & $18(24.3)$ & $30(40.5)$ & \\
\hline Moderately differentiated; Grade II & $736(23.3)$ & $191(26.0)$ & $208(28.3)$ & $337(45.8)$ & \\
\hline Poorly differentiated; Grade III & $2262(63.8)$ & $331(14.6)$ & $765(33.8)$ & $2262(63.8)$ & \\
\hline Undifferentiated; anaplastic; Grade IV & $88(2.8)$ & $18(20.5)$ & $31(35.2)$ & $39(44.3)$ & \\
\hline Nodal status, $N(\%)$ & 3309 & & & & $<0.001$ \\
\hline N0 & $431(13.0)$ & $134(31.1)$ & $95(22.0)$ & $202(46.9)$ & \\
\hline N1 & $1028(31.1)$ & $322(31.3)$ & $249(24.2)$ & $457(44.5)$ & \\
\hline $\mathrm{N} 2$ & $825(24.9)$ & $127(15.4)$ & $290(35.2)$ & $408(49.5)$ & \\
\hline N3 & $1025(31.0)$ & $40(3.9)$ & $419(40.9)$ & $566(55.2)$ & \\
\hline AJCC tumor stage & 3309 & & & & $<0.001$ \\
\hline IIB & $904(27.3)$ & $203(22.5)$ & $253(28.0)$ & $448(49.6)$ & \\
\hline IIIA & $860(26.0)$ & $255(29.7)$ & $238(27.7)$ & $367(42.7)$ & \\
\hline IIIB & $793(24.0)$ & $102(12.9)$ & $300(37.8)$ & $391(49.3)$ & \\
\hline IIIC & $752(22.7)$ & $63(8.4)$ & $262(34.8)$ & $427(56.8)$ & \\
\hline
\end{tabular}

T-stage, N-stage and AJCC tumor stage according to the 7th edition of AJCC TNM staging.

reported radiation treatment after D2-resected gastriccancerpatients can decrease recurrence and prolong survival especially patients with IIIA and IIIB patients [15]. A meta-analyses also verified that Preoperative (chemo)radiotherapy might be associated with the significant improvement in overall survival compared with over surgery alone [16]. All these studies demonstrated that radiation treatment was a potential approach to improve the outcome of selected resectable gastric cancer patients without distant metastases. Recently, the opposite result was reported there was neither survival benefit no net medical cost advantage by adding RT in Gastric Cancer adjuvant treatment [17].

However, not all resectable patients without metastatic gastric cancer obtained a survival benefit from radiation. 
Table 2: Survival rate (\%) and MST(Months) of patients with gastric cancer with distant metastases

\begin{tabular}{|c|c|c|c|c|c|c|c|c|c|c|}
\hline & \multicolumn{3}{|c|}{$\begin{array}{l}\text { Radiation prior to } \\
\text { surgery }\end{array}$} & \multicolumn{3}{|c|}{ Radiation after surgery } & \multicolumn{3}{|c|}{$\begin{array}{l}\text { No radiation and/or } \\
\text { cancer-directed surgery }\end{array}$} & \multirow[b]{3}{*}{$p$} \\
\hline & \multicolumn{2}{|c|}{$\begin{array}{l}\text { Survival } \\
\text { rate(\%) }\end{array}$} & \multirow[t]{2}{*}{ MST } & \multicolumn{2}{|c|}{ Survival rate(\%) } & \multirow[t]{2}{*}{ MST } & \multicolumn{2}{|c|}{$\begin{array}{l}\text { Survival } \\
\text { rate(\%) }\end{array}$} & \multirow[t]{2}{*}{ MST } & \\
\hline & $1 Y$ & $3 Y$ & & $1 Y$ & $3 \mathbf{Y}$ & & $1 Y$ & $3 \mathbf{Y}$ & & \\
\hline Total & 83.7 & 52.6 & 32.7 & 83.5 & 63.6 & 32.9 & 65.6 & 44.9 & 25.3 & $<0.001$ \\
\hline \multicolumn{11}{|c|}{ Age at diagnosis (y) } \\
\hline$\leq 65$ & 88.6 & 54.8 & 33.6 & 84.0 & 54.9 & 33.5 & 75.2 & 46.5 & 29.5 & $<0.001$ \\
\hline$<65$ & 77.3 & 47.3 & 30.6 & 82.7 & 50.0 & 31.8 & 58.5 & 31.8 & 22.2 & $<0.001$ \\
\hline \multicolumn{11}{|l|}{$\operatorname{Sex}, N(\%)$} \\
\hline Male & 83.0 & 53.1 & 32.3 & 84.2 & 54.5 & 33.3 & 66.5 & 39.1 & 25.7 & $<0.001$ \\
\hline Female & 86.8 & 52.0 & 32.7 & 82.5 & 50.7 & 32.2 & 64.1 & 36.3 & 24.6 & $<0.001$ \\
\hline \multicolumn{11}{|l|}{ Race, $N(\%)$} \\
\hline White & 82.7 & 50.8 & 32.1 & 82.3 & 52.7 & 32.3 & 64.5 & 38.0 & 25.1 & $<0.001$ \\
\hline Black & 90.9 & 72.7 & 33.2 & 78.8 & 41.1 & 30.0 & 60.4 & 35.8 & 23.6 & $<0.001$ \\
\hline Other & 88.3 & 70.7 & 39.9 & 89.5 & 59.5 & 35.8 & 71.6 & 38.9 & 26.5 & $<0.001$ \\
\hline \multicolumn{11}{|l|}{ T stage } \\
\hline T1a & NA & NA & NA & NA & NA & NA & NA & NA & NA & 0.191 \\
\hline $\mathrm{T} 1 \mathrm{~b}$ & NA & NA & NA & NA & NA & NA & NA & NA & NA & 0.259 \\
\hline $\mathrm{T} 2$ & 88.3 & 59.4 & 33.2 & 91.5 & 66.7 & 37.1 & 79.0 & 45.3 & 29.6 & 0.046 \\
\hline $\mathrm{T} 3$ & 85.1 & 54.2 & 33.4 & 86.0 & 56.6 & 34.3 & 73.5 & 45.2 & 28.3 & $<0.001$ \\
\hline $\mathrm{T} 4 \mathrm{a}$ & 66.0 & 28.3 & 23.1 & 79.8 & 45.3 & 30.0 & 56.8 & 28.9 & 21.5 & $<0.001$ \\
\hline $\mathrm{T} 4 \mathrm{~b}$ & 68.8 & 20.6 & 21.6 & 71.0 & 38.1 & 27.0 & 45.5 & 22.8 & 18.2 & 0.001 \\
\hline \multicolumn{11}{|l|}{ Tumor grade } \\
\hline Grade I-I & 85.2 & 53.4 & 33.7 & 85.9 & 56.0 & 35.5 & 64.5 & 38.0 & 25.1 & $<0.001$ \\
\hline Grade III-IV & 83.2 & 51.4 & 32.0 & 80.5 & 49.6 & 32.0 & 65.9 & 37.5 & 28.2 & $<0.001$ \\
\hline \multicolumn{11}{|l|}{ N-stage, $N(\%)$} \\
\hline N0 & 84.1 & 58.2 & 34.0 & 88.1 & 65.0 & 34.0 & 77.0 & 56.4 & 31.5 & 0.099 \\
\hline N1 & 85.8 & 55.9 & 33.5 & 88.1 & 68.0 & 36.9 & 79.6 & 53.1 & 31.4 & 0.001 \\
\hline $\mathrm{N} 2$ & 80.5 & 49.3 & 31.4 & 83.5 & 59.8 & 35.1 & 65.7 & 35.8 & 24.6 & $<0.001$ \\
\hline N3 & 73.9 & 11.8 & 22.4 & 80.0 & 36.4 & 28.2 & 50.6 & 19.6 & 18.2 & $<0.001$ \\
\hline \multicolumn{11}{|c|}{ AJCC tumor stage } \\
\hline IIB & 87.7 & 59.3 & 34.8 & 94.0 & 72.7 & 39.1 & 85.2 & 63.4 & 34.6 & 0.007 \\
\hline IIIA & 83.5 & 54.2 & 32.9 & 84.5 & 62.4 & 35.3 & 73.4 & 42.0 & 27.0 & $<0.001$ \\
\hline IIIB & 82.3 & 50.6 & 32.1 & 78.7 & 43.7 & 30.4 & 57.9 & 30.0 & 22.2 & $<0.001$ \\
\hline IIIC & 73.1 & 26.5 & 23.7 & 77.6 & 33.6 & 26.7 & 45.8 & 14.8 & 16.3 & $<0.001$ \\
\hline
\end{tabular}

Among those with stageT2, T4a,T4b,IIB,N0 and N1 cancer, no statistically significant differences were found in prolong survival time between RPS and no radiation groups $(p>0.05)$.

Cheng, J. et al. [18] reported that perioperative chemotherapy provided a significant improvement in OS compared to adjuvant chemoradiotherapy.
In N0 subgroup no radiation survival time is better than RPS, and no statistically significant differences were found in RAS.

INT 0116 study reported that adjuvant chemoradiotherapy after curative surgery in node negative patients provided a no significant improvement in OS (HR $=0.77,95 \% \mathrm{CI}: 0.46$ to $1.30, p=0.333$ ) [7]. In a meta- 
Table 3: Multivariate analysis (Cox Proportional Hazard Model) of overall survival for patients with gastric cancer without distant metastases

\begin{tabular}{|c|c|c|c|c|c|}
\hline & \multirow{2}{*}{ Wald } & \multirow{2}{*}{ HR } & \multicolumn{2}{|c|}{$95.0 \%$ CI for HR } & \multirow{2}{*}{$p$} \\
\hline & & & Lower & Upper & \\
\hline Gender (male) & 0.021 & 1.009 & 0.890 & 1.144 & 0.885 \\
\hline Race (white) & 20.252 & & & & $<0.001$ \\
\hline black & 12.867 & 1.349 & 1.145 & 1.588 & $<0.001$ \\
\hline Other & 18.645 & 1.608 & 1.296 & 1.995 & $<0.001$ \\
\hline Stage (IIIC) & 9.744 & & & & 0.021 \\
\hline IIB & 4.309 & 0.640 & 0.420 & 0.975 & 0.038 \\
\hline IIIA & 0.218 & 0.927 & 0.676 & 1.273 & 0.641 \\
\hline IIIB & 0.131 & 0.961 & 0.776 & 1.191 & 0.717 \\
\hline $\mathrm{T} 4 \mathrm{~b}$ & 27.569 & & & & $<0.001$ \\
\hline $\mathrm{T}(\mathrm{T} 1 \mathrm{a})$ & 2.609 & 0.371 & 0.111 & 1.236 & 0.106 \\
\hline $\mathrm{T} 1 \mathrm{~b}$ & 11.437 & 0.217 & 0.090 & 0.526 & 0.001 \\
\hline $\mathrm{T} 2$ & 11.628 & 0.477 & 0.312 & 0.730 & 0.001 \\
\hline $\mathrm{T} 3$ & 22.943 & 0.509 & 0.386 & 0.671 & $<0.001$ \\
\hline $\mathrm{T} 4 \mathrm{a}$ & 8.189 & 0.730 & 0.588 & 0.906 & 0.004 \\
\hline N (N3) & 21.272 & & & & $<0.001$ \\
\hline N0 & 11.056 & 0.509 & 0.342 & 0.758 & 0.001 \\
\hline N1 & 18.658 & 0.526 & 0.393 & 0.704 & $<0.001$ \\
\hline $\mathrm{N} 2$ & 17.035 & 0.654 & 0.534 & 0.800 & $<0.001$ \\
\hline Age $(\leq 65)$ & 51.663 & 0.644 & 0.571 & 0.726 & $<0.001$ \\
\hline Grade (I-II) & 3.632 & 0.887 & 0.785 & 1.003 & 0.057 \\
\hline Radiation (No) & 87.265 & & & & $<0.001$ \\
\hline RPS & 4.769 & 0.797 & 0.650 & 0.977 & 0.029 \\
\hline RAS & 86.933 & 0.515 & 0.448 & 0.592 & $<0.001$ \\
\hline
\end{tabular}

analysis based on 13 studies reported that OS data for node positive patients were significant benefit with the use of RT (HR $=0.73,95 \%$ CI: 0.62 to $0.86, p=0.001)$ [19].

Multivariate analysis of overall survival by Cox proportional hazards modelling, we found that the survival improvement of patients in the radiation treatment groups was significantly higher compared with that of patients in the surgery only. It reported that the 5-year survival rates were consistently longer in the postoperative chemoradiotherapy group at Stages II, IIIA, IIIB, and IV than those in the surgery only group [15].

Some limitations may have influenced the results of this study. First, our study are inherent to the methodology of retrospective analyses, including selection bias and potential confounders. Because of insufficient sample capacity we integrated the data of the following patient groups to reduce bias: Grade I and grade II were integrated into the grade I-II subgroup, Grade III and grade IV were integrated into the grade III-IV subgroup. Second, information such as the chemotherapy status, which kind surgery was performed D1 or D2 gastrectomy, locoregional recurrence, radiation techniques, total doses, fractionations, radiation-related toxicity and comorbidities were not included in the SEER database.

Finally, the determination of the T-stage, N-stage tumor stage of patients who underwent surgery depended on the postoperative pathologic results, while for those RPS patients tumor stage at diagnosis were unclear.

In conclusion, we sought to evaluate whether resectable gastric cancer patients without distant metastases would benefit from radiation. The results showed that radiation treatment was able to improve effective survival 
Table 4: Multivariate analysis (Cox Proportional Hazard Model) of overall survival of subgroups in group"RPS" group "RAS" and group "No radiation" respectively

\begin{tabular}{|c|c|c|c|c|c|c|c|c|c|c|c|c|c|c|c|}
\hline & \multicolumn{5}{|c|}{ RPS } & \multicolumn{5}{|c|}{ RAS } & \multicolumn{5}{|c|}{ No rdiation } \\
\hline & \multirow{2}{*}{ Wald } & \multirow{2}{*}{ HR } & \multicolumn{2}{|c|}{$95.0 \%$ CI for HR } & \multirow{2}{*}{$p$} & \multirow{2}{*}{ Wald } & \multirow{2}{*}{ HR } & \multicolumn{2}{|c|}{$95.0 \%$ CI for HR } & \multirow{2}{*}{$p$} & \multirow{2}{*}{ Wald } & \multirow{2}{*}{ HR } & \multicolumn{2}{|c|}{$95.0 \% \mathrm{CI}$ for $\mathrm{HR}$} & \multirow{2}{*}{$p$} \\
\hline & & & Lower & Upper & & & & Lower & Upper & & & & Lower & Upper & \\
\hline Gender (male) & 0.138 & 1.090 & 0.693 & 1.714 & 0.710 & 0.098 & 0.962 & 0.757 & 1.224 & 0.754 & 0.008 & 0.993 & 0.848 & 1.163 & 0.930 \\
\hline Race (white) & 2.940 & & & & 0.230 & 12.825 & & & & 0.002 & 9.839 & & & & 0.007 \\
\hline black & 1.176 & 1.586 & 0.689 & 3.651 & 0.278 & 6.732 & 1.494 & 1.103 & 2.023 & 0.009 & 5.540 & 1.274 & 1.041 & 1.558 & 0.019 \\
\hline Other & 0.376 & 0.597 & 0.115 & 3.099 & 0.540 & 12.509 & 1.968 & 1.352 & 2.864 & $<0.001$ & 9.374 & 1.525 & 1.164 & 1.998 & 0.002 \\
\hline Stage (IIIC) & 3.563 & & & & 0.313 & 5.014 & & & & 0.171 & 5.744 & & & & 0.125 \\
\hline IIB & 1.448 & 0.331 & 0.055 & 2.005 & 0.229 & 2.925 & 0.469 & 0.197 & 1.117 & 0.087 & 2.614 & 0.648 & 0.383 & 1.096 & 0.106 \\
\hline IIIA & 0.110 & 0.818 & 0.250 & 2.676 & 0.740 & 0.698 & 0.759 & 0.398 & 1.449 & 0.403 & 0.103 & 0.937 & 0.630 & 1.394 & 0.748 \\
\hline IIIB & 0.037 & 0.898 & 0.301 & 2.683 & 0.848 & 0.068 & 0.947 & 0.630 & 1.425 & 0.794 & 0.090 & 0.960 & 0.736 & 1.253 & 0.764 \\
\hline $\mathrm{T}(\mathrm{T} 4 \mathrm{~b})$ & 7.420 & & & & 0.191 & 3.217 & & & & 0.667 & 19.409 & & & & 0.002 \\
\hline T1a & 0.992 & 2.625 & 0.393 & 17.532 & 0.319 & 0.004 & 0.000 & 0.000 & 7.300 & 0.950 & 0.015 & 0.000 & 0.000 & 2.470 & 0.904 \\
\hline $\mathrm{T} 1 \mathrm{~b}$ & 0.003 & 0.000 & 0.000 & 15.070 & 0.957 & 2.162 & 0.305 & 0.063 & 1.485 & 0.141 & 6.988 & 0.231 & 0.078 & 0.684 & 0.008 \\
\hline $\mathrm{T} 2$ & 0.009 & 1.074 & 0.236 & 4.899 & 0.926 & 1.023 & 0.630 & 0.258 & 1.541 & 0.312 & 9.566 & 0.426 & 0.248 & 0.732 & 0.002 \\
\hline $\mathrm{T} 3$ & 1.123 & 0.579 & 0.211 & 1.591 & 0.289 & 1.796 & 0.676 & 0.381 & 1.198 & 0.180 & 17.756 & 0.480 & 0.341 & 0.676 & $<0.001$ \\
\hline $\mathrm{T} 4 \mathrm{a}$ & 0.086 & 0.852 & 0.293 & 2.480 & 0.769 & 0.424 & 0.868 & 0.567 & 1.328 & 0.515 & 8.092 & 0.681 & 0.523 & 0.887 & 0.004 \\
\hline $\mathrm{N}(\mathrm{N} 3)$ & 2.345 & & & & 0.504 & 4.165 & & & & 0.244 & 14.814 & & & & 0.002 \\
\hline N0 & 0.090 & 1.322 & 0.214 & 8.153 & 0.764 & 0.466 & 0.746 & 0.322 & 1.729 & 0.495 & 9.652 & 0.462 & 0.284 & 0.752 & 0.002 \\
\hline N1 & 0.258 & 0.740 & 0.231 & 2.371 & 0.612 & 0.516 & 0.804 & 0.443 & 1.458 & 0.472 & 14.215 & 0.492 & 0.340 & 0.712 & $<0.001$ \\
\hline N2 & 0.314 & 0.733 & 0.247 & 2.171 & 0.575 & 3.657 & 0.689 & 0.470 & 1.009 & 0.056 & 9.112 & 0.675 & 0.523 & 0.871 & 0.003 \\
\hline Age $(\leq 65)$ & 5.084 & 0.669 & 0.472 & 0.949 & 0.024 & 0.875 & 0.894 & 0.708 & 1.130 & 0.350 & 52.995 & 0.552 & 0.470 & 0.648 & $<0.001$ \\
\hline Grade (I-II) & 1.552 & 0.807 & 0.576 & 1.131 & 0.213 & 2.196 & 0.839 & 0.665 & 1.058 & 0.138 & 0.893 & 0.924 & 0.786 & 1.088 & 0.345 \\
\hline
\end{tabular}

time in T3, T4a, T4b, N1, N2, N3, IIIA, IIIB and IIIC cancer. RPS is different from RAS in special subgroup.

From the results of this study, we considered that patients who were younger and those with locally advanced stage primary tumors might obtain a greater survival benefit from radiation treatment than others.

Additionally, radiation therapy may strengthen the survival benefit that is gained from surgical treatment. Our study was a retrospective analysis with limitations and further prospective randomized trial are needed to validate our hypothesis.

\section{MATERIALS AND METHODS}

\section{Patient selection}

The Surveillance, Epidemiology, and End Results Program (SEER) database is an authoritative source of information on cancer incidence and survival, sponsored by the National Cancer Institute. In the SEER database, currently collects and publishes cancer incidence and survival data from 18 population-based cancer registries, which covers approximately 28 percent of the population in the United States.

The database of the SEER program includes information on patient demographics, race, sex, tumor histology, primary tumor site, stage at initial diagnosis, surgery, radiotherapy, and survival.

Our retrospective study contained 5744 patients from the SEER database (SEER*Stat 8.3.4) who were initially diagnosed with histologically confirmed gastric cancer without distant metastases, between 2010 and 2013. The characteristics of 5744 patients with gastric cancer without distant metastases in Supplementary Table 1. Histological type were limited to adenocarcinoma, mucinous adenocarcinoma, signet ring cell carcinoma. Inclusion criteria included only patients who underwent D2 surgical treatment with age of diagnosis more than 18 years; one primary only; cause of death dead due to cancer; surgery performed; without distant metastases; AJCC's TNM stage of the 7th edition. Exclusion criteria included patients less than 18 years of age; distant metastases and occult gastric cancer (no evidence of primary tumor). The IA, IB, IIA subgroups were eliminated considering the far smaller number 

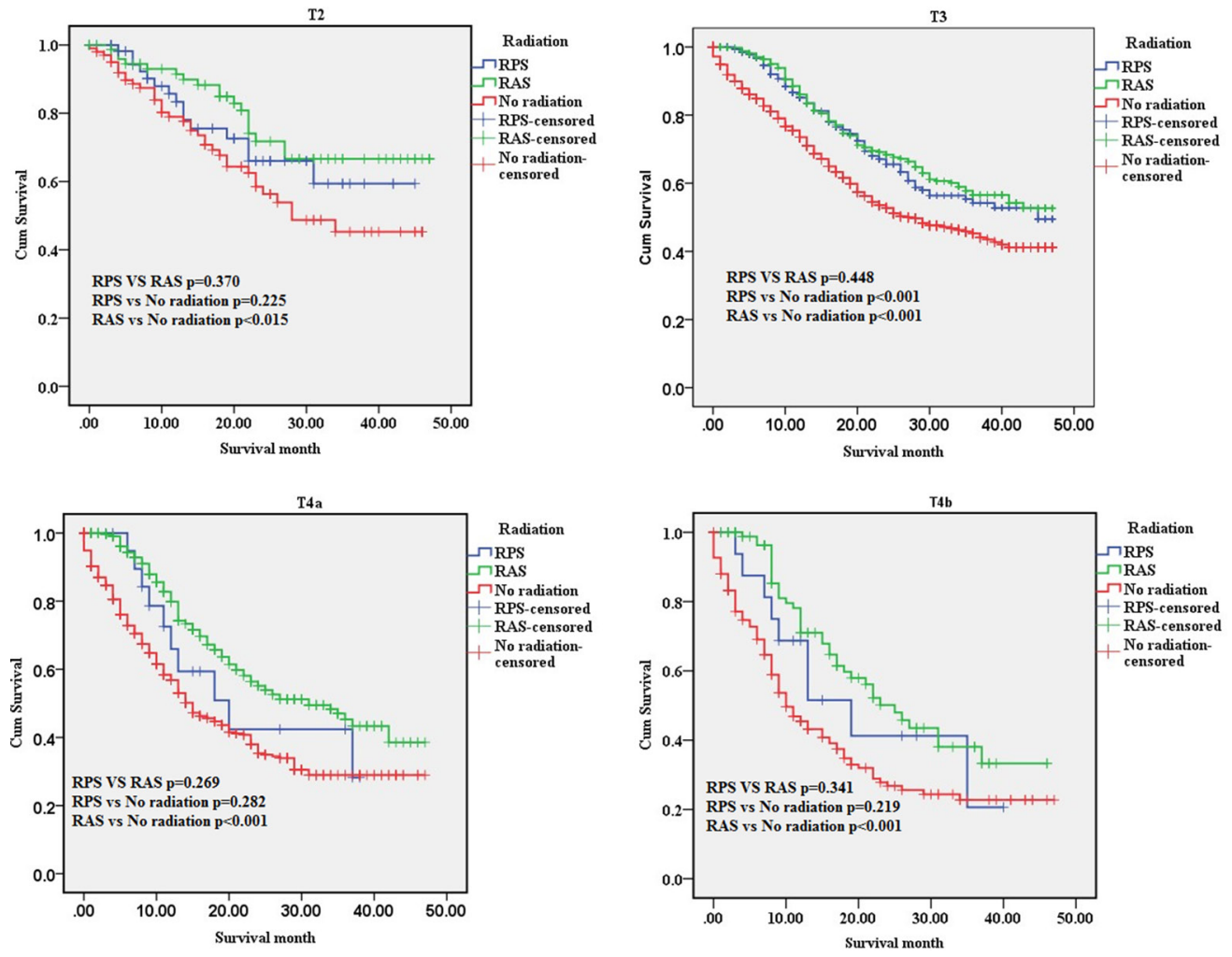

Figure 1: The survival curves of three groups in T-stage subgroups. T2, T3, T4a and T4b subgroup. RPS: patients who underwent radiation after surgery; RAS: patients who underwent radiation prior to surgery; No radiation: patients did not undergo any radiation.
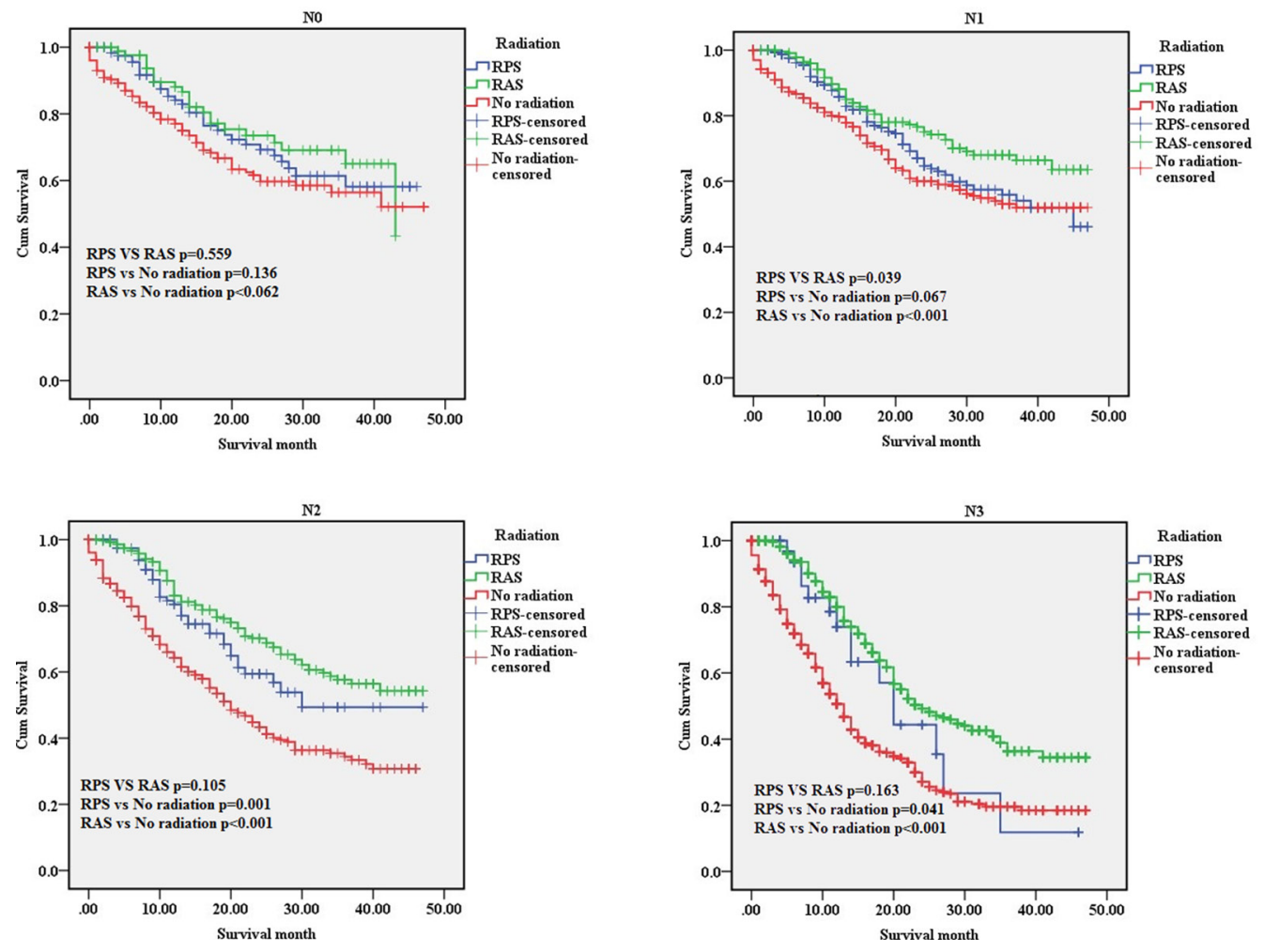

Figure 2: The survival curves of three groups in N-stage subgroups. N0 subgroup; N1 subgroup; N2 subgroup; N3 subgroup. RPS: patients who underwent radiation after surgery; RAS: patients who underwent radiation prior to surgery; No radiation: patients did not undergo any radiation. 

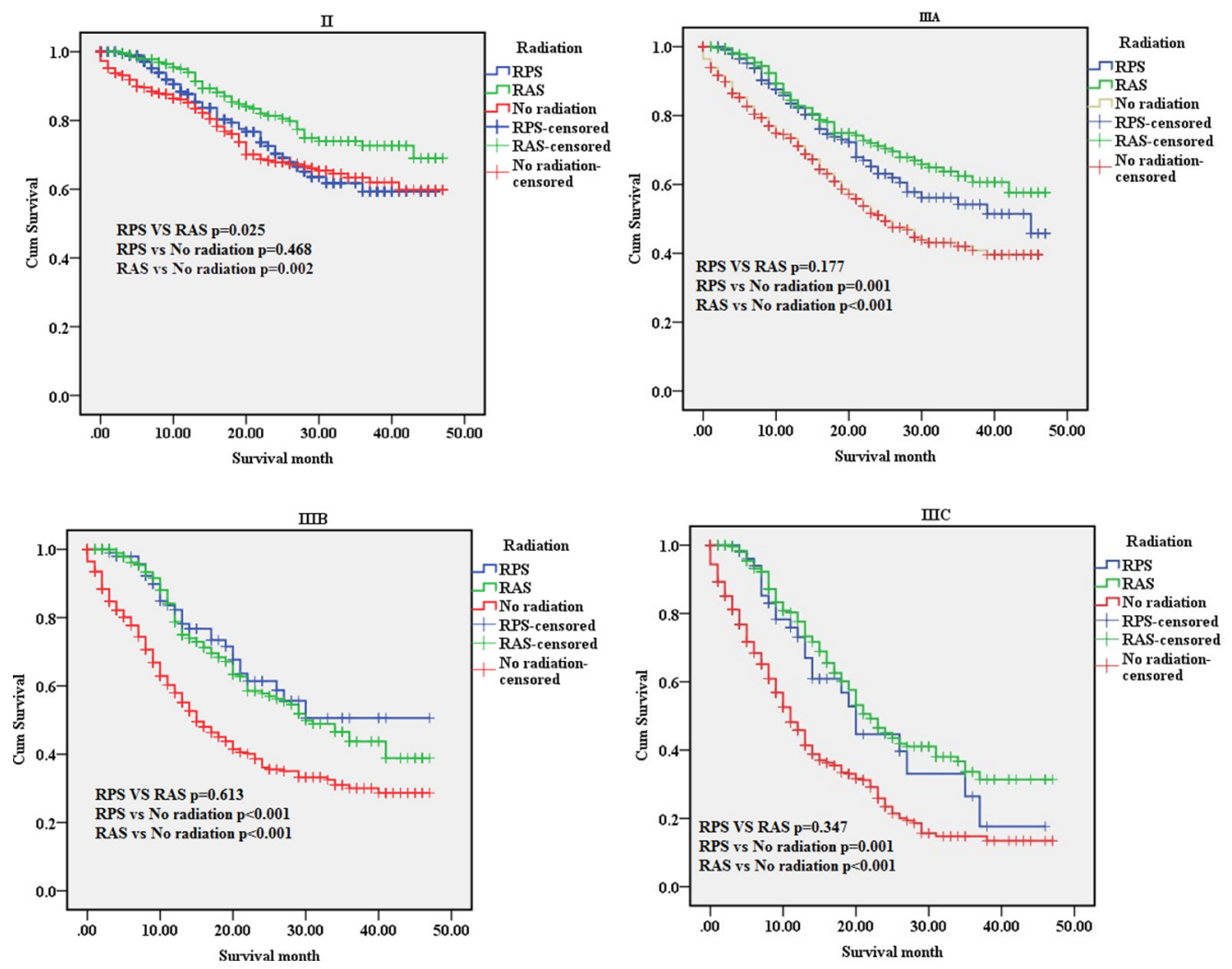

Figure 3: The survival curves of three groups in tumor stage subgroups. II subgroup; IIIA subgroup; IIIB subgroup; IIIC subgroup. RPS: patients who underwent radiation after surgery; RAS: patients who underwent radiation prior to surgery; No radiation: patients did not undergo any radiation.

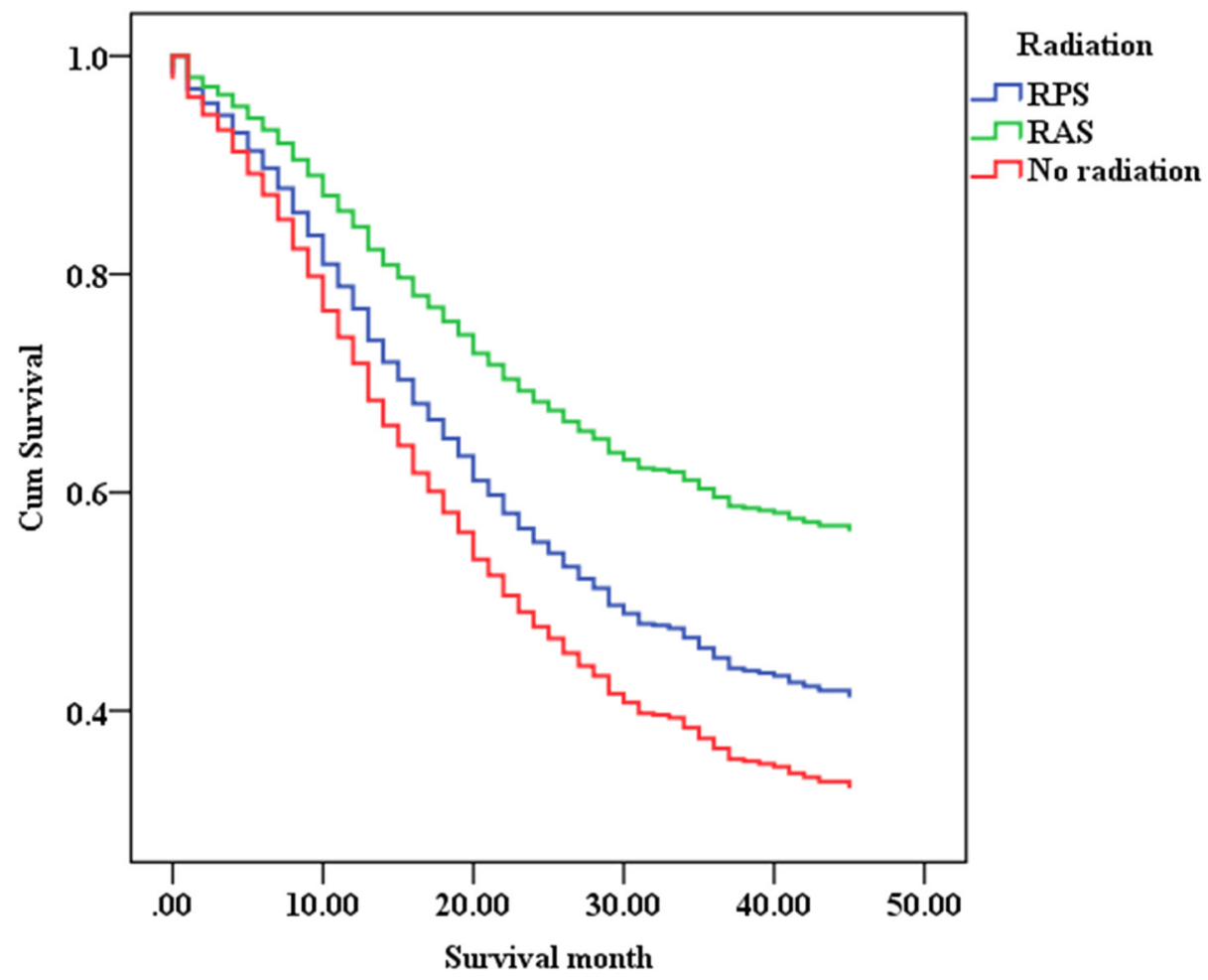

Figure 4: The multivariate survival curves of gastric cancer without distant metastases in different groups. 
of patients who underwent radiation. The remaining 3309 patients were divided into patients who underwent radiation after surgery ("RAS" group) and patients who underwent radiation prior to surgery ("RPS" group).

\section{Statistical analysis}

Count data were analyzed by chi-square test. The median survival time (MST) and survival curves, were estimated with the Kaplan-Meier method, and the log-rank test was performed to evaluate survival in the different groups. Hazard ratios (HRs) along with 95\% confidence intervals (CI) were calculated using the multivariate Cox proportional hazard regression model to determine the influences of other factors including age, race, gender, grade, histological type, T-stage, and N-stage, on survival. Statistical tests were two-sided, $p<0.05$ was considered statistically significant. SPSS22.0 (SPSS Chicago, IL, USA) software was used for the statistical analysis.

\section{CONFLICTS OF INTEREST} interests.

The authors declare that they have no competing

\section{FUNDING}

This study was not supported by any pharmaceutical corporation or company; the publication fee was paid by the authors' institution.

\section{REFERENCES}

1. Parkin DM, Pisani P, Ferlay J. Global cancer statistics. CA Cancer J Clin. 2011; 61:87.

2. Wang J, Sun Y, Bertagnolli MM. Comparison of gastric cancer survival between Caucasian and Asian patients treated in the United States: results from the Surveillance Epidemiology and End Results (SEER) database. Ann Surg Oncol. 2015; 22:2965-2971.

3. Siegel RL, Miller KD, Jemal A. Cancer statistics, 2016. CA Cancer J Clin. 2016; 66:7.

4. Ferlay J, Soerjomataram I, Dikshit R, Eser S, Mathers C, Rebelo M, Parkin DM, Forman D, Bray F. Cancer incidence and mortality worldwide: sources, methods and major patterns in GLOBOCAN 2012. Int J Cancer. 2015; 136:E359.

5. Pătraşcu F, Croitoru A, Gramaticu I, Andrei M. Locally advanced or metastatic gastric cancer - an epidemiological and diagnosing update. Jurnalul De Chirurgie. 2011; 7.

6. Yang Q, Wei Y, Chen YX, Zhou SW, Jiang ZM, Xie DR. Indirect comparison showed survival benefit from adjuvant chemoradiotherapy in completely resected gastric cancer with d2 lymphadenectomy. Gastroent Res Pract. 2013; 2013:634929.

7. Macdonald JS, Smalley SR, Benedetti J, Hundahl SA, Estes NC, Stemmermann GN, Haller DG, Ajani JA,
Gunderson LL, Jessup JM. Chemoradiotherapy after surgery compared with surgery alone for adenocarcinoma of the stomach or gastroesophageal junction. N Engl J Med. 2001; 345:725-730.

8. Soon YY, Leong CN, Tey JC, Tham IW, Lu JJ. Postoperative chemo-radiotherapy versus chemotherapy for resected gastric cancer: a systematic review and meta-analysis. J Med Imag Radiat On. 2014; 58:483.

9. Yu J, Lim DH, Ahn YC, Lee J, Kang WK, Park SH, Park JO, Park YS, Lim HY, Kim ST. Effects of adjuvant radiotherapy on completely resected gastric cancer: A radiation oncologist's view of the ARTIST randomized phase III trial. Radiother Oncol. 2015; 117:171-177.

10. Lee JA, Yong CA, Lim DH, Park HC, Asranbaeva MS. Dosimetric and Clinical Influence of 3D Versus 2D Planning in Postoperative Radiation Therapy for Gastric Cancer. Cancer Res Treat. 2015; 47:727.

11. Park JM, Kim YH. Current approaches to gastric cancer in Korea. Gastrointest cancer res. 2008; 2:137-144.

12. Sano T, Aiko T. New Japanese classifications and treatment guidelines for gastric cancer: revision concepts and major revised points. Gastric Cancer. 2011; 14:97-100.

13. Lim DH. Postoperative Adjuvant Radiotherapy for Patients with Gastric Adenocarcinoma. J Gastric Cancer. 2012; 12:205-209.

14. Valentini V, Cellini F, Minsky BD, Mattiucci GC, Balducci M, D'Agostino G, D'Angelo E, Dinapoli N, Nicolotti N, Valentini C, La Torre G. Survival after radiotherapy in gastric cancer: systematic review and meta-analysis. Radiother Oncol. 2009; 92:176-183.

15. Kim S, Lim DH, Lee J, Kang WK, Macdonald JS, Park CH, Park SH, Lee SH, Kim K, Park JO. An observational study suggesting clinical benefit for adjuvant postoperative chemoradiation in a population of over 500 cases after gastric resection with D2 nodal dissection for adenocarcinoma of the stomach. Int J Radiat Oncol. 2005; 63:1279-1285.

16. Kumagai K, Rouvelas I, Tsai JA, Mariosa D, Lind PA, Lindblad M, Ye W, Lundell L, Schuhmacher C, Mauer M. Survival benefit and additional value of preoperative chemoradiotherapy in resectable gastric and gastrooesophageal junction cancer: a direct and adjusted indirect comparison meta-analysis. Eur J Surg Oncol. 2015; 41:282.

17. Chang JS, Choi Y, Shin J, Kim KH, Keum KC, Kim HS, Koom WS, Park EC. Patterns of Care for Radiotherapy in the Neoadjuvant and Adjuvant Treatment of Gastric Cancer: A Twelve-Year Nationwide Cohort Study in Korea. Cancer Res Treat. 2017 Mar 8. [Epub ahead of print].

18. Cheng J, Squires MH 3rd, Mikell JL, Fisher SB, Staley CA 3rd, Kooby DA, El-Rayes BF, Curran WJ Jr, Hall WA, Colbert LE, Shelton JW, Maithel SK, Landry J, Yu DS. Radiotherapy patterns of care in gastric adenocarcinoma: a single institution experience. J Gastrointest Oncol. 2015; 6:247-253.

19. Ohri N, Garg MK, Aparo S, KaubischA, Tome W, Kennedy TJ, Kalnicki S, Guha C. Who benefits from adjuvant radiation therapy for gastric cancer? A meta-analysis. Int J Radiat Oncol. 2013; 86:330-335. 